\title{
Compatibility and Crystallization Studies on Poly(phenyl acetylene)/Polycaprolactone Blend
}

\author{
Hsin-Lung Chen, ${ }^{* \dagger}$ Der-Jang Liaw, Been-Yang Liaw, Chu-Lun ShiH, \\ and Jang-Shiang TsaI \\ Department of Chemical Engineering, National Taiwan University of Science and Technology, \\ Taipei, Taiwan, Republic of China \\ * Department of Chemical Engineering, National Tsing Hua University, \\ Hsin-Chu, Taiwan 30034, Republic of China
}

(Received January 16, 1998)

\begin{abstract}
The compatibility and crystallization behavior of poly(phenyl acetylene) (PPA)/polycaprolactone (PCL) blends were investigated. PPA and PCL were partially miscible in the melt. PCL composition in the PCL-rich phase was calculated from the observed glass transition temperature with the consideration of the perturbation of amorphous phase composition by the crystallization of PCL. PCL composition in PCL-rich phase ranged from $c a .91 \mathrm{wt} \%$ to $94 \mathrm{wt} \%$. The melting point of PCL was depressed upon blending. The isothermal crystallization kinetics of the blends was analyzed by Avrami equation. The crystallization rate decreased with increasing PPA composition in the PCL-rich phase. The crystal fold surface free energy $\sigma_{\mathrm{e}}$ of $c a .90 \mathrm{erg} \mathrm{cm}^{-2}$ was obtained from Lauritzen-Hoffman analysis considering regime II kinetics. Fold surface free energy was found to increase slightly with PPA composition. KEY WORDS Poly(phenyl acetylene) / Polycaprolactone / Blend / Compatibility / Crystallization /
\end{abstract}

Blending is an effective method for tailoring the properties of polymers. Thermal, mechanical, and rheological properties may all be controlled by polymer blending. Recently, we have been interested in the blends of polycaprolactone (PCL) with several polymers containing conjugated double bonds in the backbone. ${ }^{1} \mathrm{~A}$ conjugated structure may impart conductive properties to the polymers. In spite of the conductive functionality, conducting polymers usually suffer the disadvantage of not being easily processed, especially for melt processing. It is therefore of interest to blend these materials with conventional thermoplastics to overcome this drawback.

A previous study investigated the miscibility of the binary blends of polystyrene (PS) and PCL with the alternating copolymer of phenyl acetylene (PA) and carbon monoxide (poly(PA-alt-CO)). ${ }^{1}$ These bindary systems were miscible in the amorphous state and crystallization rate of PCL was depressed by the miscibility. In addition to the copolymer of PA and $\mathrm{CO}$, blends with poly(phenylene acetylene) (PPA) homopolymer are also of interest. PPA is also a conjugated polymer which has been polymerized via radical, ${ }^{2-4}$ cationic, ${ }^{5,6}$ or coordination mechanism. ${ }^{7-12}$ Only oligomers with low molecular weight ( $c a .1000)$ were formed by radical or cationic mechanism. Masuda et al. reported that PA is polymerized to high molecular weight by tungsten or molybdenum. ${ }^{13-19}$ Recently, Liaw et al. reported the polymerization of PA initiated by tungsten carbene complex. ${ }^{20}$ PPA thus obtained had molecular weight above 10000.

In this study, we blended PPA polymerized by tungsen carbene complex with PCL. The compatibility of this binary combination will be discussed. The effects of compatibility on crystallization of PCL is described.

\footnotetext{
† To whom correspondence should be addressed.
}

\section{EXPERIMENTAL}

PPA was synthesized by tungsten carbene complex. The synthetic scheme and method have been reported in the previous publication. ${ }^{20}$ The molecular weights of PPA were $M_{n}=12000$ and $M_{w}=15000$. PCL with molecular weight around 10000 was obtained from YunJian Polymer Inc. Ltd., Taiwan.

Blending of PPA with PCL was carried out by solution casting. The blending components were dissolved in chloroform at room temperature yielding a $1 \mathrm{wt} \%$ solution. The blend was obtained after evaporating the solvent in a rotatory evaporator at $80^{\circ} \mathrm{C}$. The blend was further dried in vacuo at $60^{\circ} \mathrm{C}$ for $24 \mathrm{~h}$.

Thermal analysis of the blends was performed with a TA Instrument 2000 differential scanning calorimeter. For $T_{\mathrm{g}}$ measurement, the sample was first annealed at $135^{\circ} \mathrm{C}$ for $3 \mathrm{~min}$ followed by quenching into liquid nitrogen. The sample was subsequently scanned at $20^{\circ} \mathrm{C} \mathrm{min}^{-1}$ from $-100^{\circ} \mathrm{C}$ to $140^{\circ} \mathrm{C}$ to record the glass transition region. For measurement of melting point, the sample was annealed at $135^{\circ} \mathrm{C}$ for 3 min followed by cooling at $c a .100^{\circ} \mathrm{Cmin}^{-1}$ to the desired crystallization temperatures. DSC scanning was conducted at $20^{\circ} \mathrm{C} \mathrm{min}^{-1}$ after $2 \mathrm{~h}$ of crystallization. For the study of isothermal crystallization kinetics, the sample was annealed at $135^{\circ} \mathrm{C}$ for $3 \mathrm{~min}$ followed by cooling at $c a$. $100^{\circ} \mathrm{C} \mathrm{min}^{-1}$ to the crystallization temperature, where the isothermal crystallization exotherm was recorded. The morphologies of PCL/PPA blends in the melt were observed by a Hund Wetzlar cross-polarized optical microscopy. The blends were melted at $135^{\circ} \mathrm{C}$ on a Linkam HFS91 hot stage where the melt morphologies were observed.

\section{RESULTS AND DISCUSSION}

The compatibility between PPA and PCL was evaluated by observing glass transition temperatures. Figure 1 displays the DSC thermograms for the glass 


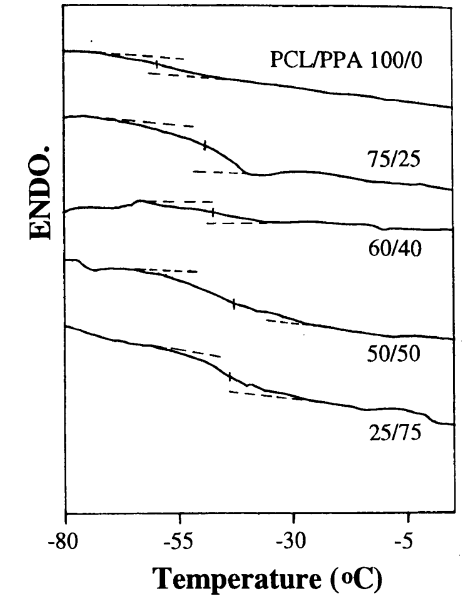

Figure 1. DSC thermograms of the glass transition regions of PCL/PPA blends quenched from $135^{\circ} \mathrm{C}$.

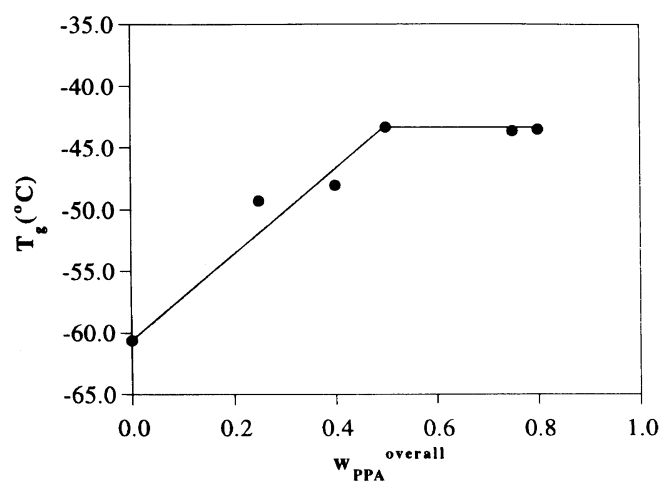

Figure 2. $T_{\mathrm{g}}$ vs. overall PPA composition plots.

transition regions of PCL/PPA blends quenched from $135^{\circ} \mathrm{C}$. The $T_{g}$ of pure PCL is $-60^{\circ} \mathrm{C}$. $T_{\mathrm{g}}$ moves upward to $-49^{\circ} \mathrm{C}$ when PCL was blended with $25 \mathrm{wt} \%$ PPA. Rise in $T_{\mathrm{g}}$ indicates the presence of segmental mixing between PCL and PPA. Figure 2 plots the observed $T_{\mathrm{g}}$ against the overall composition of PPA. $T_{\mathrm{g}}$ increases to $-43^{\circ} \mathrm{C}$ then levels off at the overall PPA composition, $w_{\text {PPA }}^{\text {overall }}=0.5$. Although elevation in $T_{\mathrm{g}}$ may indicate segmental mixing between PPA and PCL, it appears that these two polymers are not completely miscible because full miscibility should lead to a monotonic increase in $T_{\mathrm{g}}$ with overall PPA composition.

To evaluate the compatibility in further detail, the morphologies of PCL/PPA blends in the melt were observed by optical microscopy. Figure 3 displays the morphology of PCL/PPA 75/25 and 50/50 blends. Phase-separated morphology was clearly identified with the dark regions corresponding to the PPA-rich phase and bright regions corresponding to the PCL-rich phase. PCL and PPA were hence not fully miscible but exhibited partial miscibility in the melt. The $T_{\mathrm{g}} \mathrm{s}$ observed in Figure 1 should correspond to the glass transitions of the PCL-rich phase.

The extent of partial miscibility is determined by the compositions of the two phases. The composition of the PCL-rich phase may in principle be estimated from the observed $T_{\mathrm{g}}$ through the application of Fox equation, which is usually used for blends with random mixing ${ }^{21}$ :

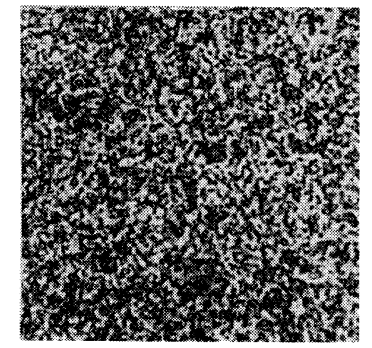

(a) PCL/PPA 75/25

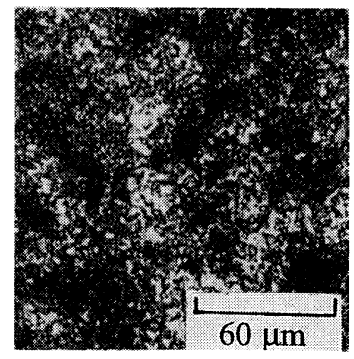

(b) $50 / 50$
Figure 3. Phase-separated morphology of PCL/PPA (a) $75 / 25$ and (b) $50 / 50$ blends at $135^{\circ} \mathrm{C}$.

$$
\frac{1}{T_{\mathrm{g}}}=\frac{\left(1-w_{2}\right)}{T_{\mathrm{g} 1}}+\frac{w_{2}}{T_{\mathrm{g} 2}}
$$

where $w_{2}$ is the weight fraction of PCL in the mixed phase, and $T_{\mathrm{g} 1}=121^{\circ} \mathrm{C}$ and $T_{\mathrm{g} 2}=-60^{\circ} \mathrm{C}$, the glass transition temperatures of pure PPA and PCL, respectively. Rearranging eq 1 , the PCL composition in the PCL-rich phase corresponding to the observed $T_{\mathrm{g}}$ can be calculated by

$$
w_{2}=\frac{1}{\left(T_{\mathrm{g} 1}-T_{\mathrm{g} 2}\right)}\left(\frac{T_{\mathrm{g} 1} T_{\mathrm{g} 2}}{T_{\mathrm{g}}}-T_{\mathrm{g} 2}\right)
$$

However, it is noted that the blends were actually semicrystalline prior to $T_{\mathrm{g}}$ measurements. This was manifested by the presence of a melting endotherm of PCL in the DSC scan. PCL crystals should be primarily formed within the PCL-rich phase. The crystallization of PCL in the PCL-rich phase may disrupt the composition of the amorphous phase and thus the amorphous phase composition corresponding to the observed $T_{\mathrm{g}}$ should deviate from the actual composition in the PCL-rich phase. Assuming that all PCL crystals were formed in the PCL-rich phase, the actual PCL composition in the PCL-rich phase $\left(w_{\mathrm{PCL}}^{\prime}\right)$ is given by

$$
w_{\mathrm{PCL}}^{\prime}=\frac{W_{\mathrm{PCL}-\text { rich }}^{\mathrm{a}} w_{\mathrm{PCL}}^{\mathrm{a}}+W_{\mathrm{c}}}{W_{\mathrm{PCL}-\text { rich }}}
$$

where $W_{\mathrm{PCL}-\text { rich }}^{\mathrm{a}}$ is the weight of the amorphous portion in the PCL-rich phase, $w_{\mathrm{PCL}}^{\mathrm{a}}$ is the composition of PCL in the amorphous portion in the PCL-rich phase (this is just the composition derived from the observed $T_{\mathrm{g}}$ through eq 2), $W_{\text {PCL-rich }}$ is the weight of PCL-rich phase, and $W_{\mathrm{c}}$ is the weight of PCL crystals. Note that

$$
W_{\mathrm{PCL}-\text { rich }}^{\mathrm{a}}=W_{\mathrm{PCL}-\mathrm{rich}}-W_{\mathrm{c}}
$$

Inserting eq 4 into eq 3 , we have,

$$
w_{\mathrm{PCL}}^{\prime}=w_{\mathrm{PCL}}^{\mathrm{a}}+\frac{W_{\mathrm{c}}\left(1-w_{\mathrm{PCL}}^{\mathrm{a}}\right)}{W_{\mathrm{PCL}-\text { rich }}}
$$

Since $w_{\mathrm{PCL}}^{\mathrm{a}}$ can be evaluated from the observed $T_{\mathrm{g}}$ and $W_{\mathrm{c}}$ is obtained from the observed enthalpy of melting, $w_{\mathrm{PCL}}^{\prime}$ can be calculated once the weight of PCL-rich phase, $W_{\text {PCL-rich }}$, is known. $W_{\text {PCL-rich }}$ may be evaluated assuming the crystallizability of PCL in the PCL-rich phase is essentially unaffected by the slight dilution of PPA. Figure 4 plots the overall degree of crystallinity $\left(=W_{\mathrm{c}} / W\right.$, $W$ is the total weight of the blend) against the overall PCL composition ( $\left.w_{\mathrm{PCL}}^{\text {overall }}\right)$. A good linear rule is found 


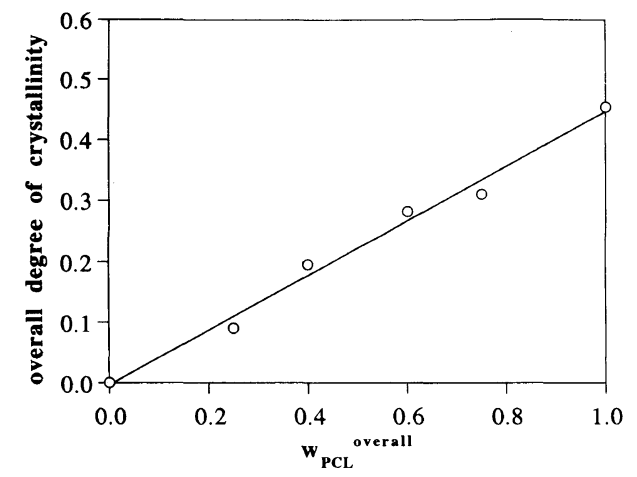

Figure 4. Overall degree of crystallinity $v s$. overall PCL composition.

in the plot, indicating that the weight of PCL crystal can be expressed as

$$
W_{\mathrm{c}}=W w_{\mathrm{c}}^{0} w_{\mathrm{PCL}}^{\mathrm{overall}}
$$

where $w_{\mathrm{c}}^{0}$ is the degree of crystallinity of pure PCL. The crystallizability of PCL in PCL-rich phase is expressed by the weight of PCL crystals normalized by the total weight of PCL in PCL-rich phase, namely

$$
\text { crystallizability }=\frac{W_{\mathrm{c}}}{W_{\mathrm{PCL}-\mathrm{rich}} w_{\mathrm{PCL}}^{\prime}}
$$

Substituting eq 6 into eq 7 to yield

$$
\text { crystallizability }=\frac{W w_{\mathrm{c}}^{0} w_{\mathrm{PC}}^{\text {overall }}}{W_{\mathrm{PCL}-\text { rich }} w_{\mathrm{PCL}}^{\prime}}
$$

If crystallizability of PCL in PCL-rich phase is the same as that of pure PCL, then

$$
\frac{W w_{\mathrm{c}}^{0} w_{\mathrm{PCL}}^{\text {overall }}}{W_{\mathrm{PCL}-\text { rich }} w_{\mathrm{PCL}}^{\prime}}=w_{\mathrm{c}}^{0}
$$

The weight of PCL-rich phase is thus given by,

$$
W_{\mathrm{PCL}-\text { rich }}=\frac{W w_{\mathrm{PCL}}^{\mathrm{overall}}}{w_{\mathrm{PCL}}^{\prime}}
$$

Inserting eq 10 into eq 5 , the actual composition of PCL in PCL-rich phase is,

$$
w_{\mathrm{PCL}}^{\prime}=\frac{w_{\mathrm{PCL}}^{\mathrm{a}}}{1-w_{\mathrm{c}}^{0}\left(1-w_{\mathrm{PCL}}^{\mathrm{a}}\right)}
$$

Table I summarizes the parameters defined in the derivation of eq 11. The calculated PCL compositions are listed in Table II. The PCL composition in PCL-rich phase ranges from $c a .91 \mathrm{wt} \%$ to $94 \mathrm{wt} \%$. The composition increases with overall PCL composition but levels off at $91 \mathrm{wt} \%$ when overall PCL composition exceeds $60 \mathrm{wt} \%$. This appears to contradict the phase diagram which prescribes that the PCL-rich phase should have the same equilibrium composition irrespective of overall blend composition. This is by no means due to the uncertainty associated with the evaluation of PCL composition by eq 11. As shown later, crystallization rate and melting point of PCL drop with decreasing

\begin{tabular}{|c|c|}
\hline Parameter & Definition \\
\hline$w_{\mathrm{PCL}}^{\prime}$ & Actual PCL composition in PCL-rich phase \\
\hline$W_{\mathrm{PCL}-\text { rich }}^{\mathrm{a}}$ & Weight of amorphous portion in PCL-rich phase \\
\hline$w_{\mathrm{PCL}}^{\mathrm{a}}$ & $\begin{array}{l}\text { Composition of PCL in the amorphous portion in the } \\
\text { PCL-rich phase }\end{array}$ \\
\hline$W_{\text {PCL-rich }}$ & Weight of PCL-rich phase \\
\hline$W_{\mathrm{c}}$ & Weight of PCL crystals \\
\hline$W^{c}$ & Total weight of the blend \\
\hline$w_{\mathrm{c}}^{0}$ & Degree of crystallinity of pure PCL \\
\hline$w_{\mathrm{PCL}}^{\mathrm{overa}}$ & Overall composition of PCL \\
\hline
\end{tabular}
overall PCL composition. Such depression is consistent with a lower PCL composition in the PCL-rich phase in the blend with lower overall PCL content. The contradiction is alternatively ascribed to the possibility that
Table I. Parameters used for derivation of eq 11

Table II. Composition of PCL in the PCL-rich phase calculated from eq 11

\begin{tabular}{ll}
\hline$w_{\mathrm{PCL}}^{\text {overall }}$ & $w_{\mathrm{PCL}}^{\prime}$ \\
\hline 0.75 & 0.94 \\
0.60 & 0.93 \\
0.50 & 0.91 \\
0.25 & 0.92 \\
0.20 & 0.91 \\
\hline
\end{tabular}

the observed composition of PCL-rich phase is not the equilibrium value. The compositions were deduced from the observed $T_{\mathrm{g}}$ measured after annealing the as-prepared blend at $135^{\circ} \mathrm{C}$ for $3 \mathrm{~min}$, and thus this melt annealing may not be sufficient to bring the two phases to their equilibrium compositions predicted by the binodal lines. One reason is that $135^{\circ} \mathrm{C}$ is only slightly higher than the $T_{\mathrm{g}}$ of PPA-rich phase. Attainment of equilibrium compositions requires transport of PCL and PPA molecules from two phases. The diffusion of PPA or PCL molecules into PPA-rich phase may be hindered due to the high viscosity of PPA-rich phase. Similarly, diffusion of PCL or PPA out of the PPA-rich phase may also be difficult due to high matrix viscosity. The equilibrium compositions may thus not be reached after annealing at $135^{\circ} \mathrm{C}$ for $3 \mathrm{~min}$. Attainment of the equilibrium compositions may certainly be facilitated by annealing for a significantly longer time or at a higher temperature. However, because PPA may undergo crosslinking reactions with prolonged annealing at temperatures above its $T_{\mathrm{g}}$, attempt was not made to further increase the annealing time and temperature.

Our previous study found that PCL was miscible with poly(PA-alt-CO). ${ }^{1}$ As the chemical structure of PA differs from that of the copolymer by a carbonyl group per repeating unit, the $\mathrm{CO}$ group in the copolymer may interact favorably with PCL, which consequently led to the miscibility. Another reason for the miscibility is that the presence of $\mathrm{CO}$ group imparts more conformational freedom to the polymer chain. PPA has a poorer miscibility with other polymers due to a stiffer chain.

Figure 5 displays the effect of blending on the melting point of PCL. The observed melting point is plotted against PCL composition in PCL-rich phase. The melting point of PCL decreases with increasing PPA content. Because complete miscibility was absent, the equilibrium melting point of PCL cannot be depressed by blending with PPA. Depression of the observed melting point (which corresponds to the nonequilibrium melting point) 


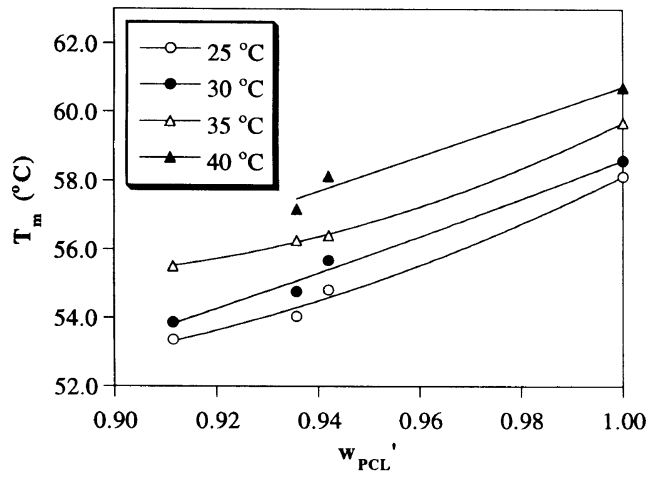

Figure 5. Variation in melting point with composition of PCL in the PCL-rich phase. Corresponding crystallization temperatures are indicated.

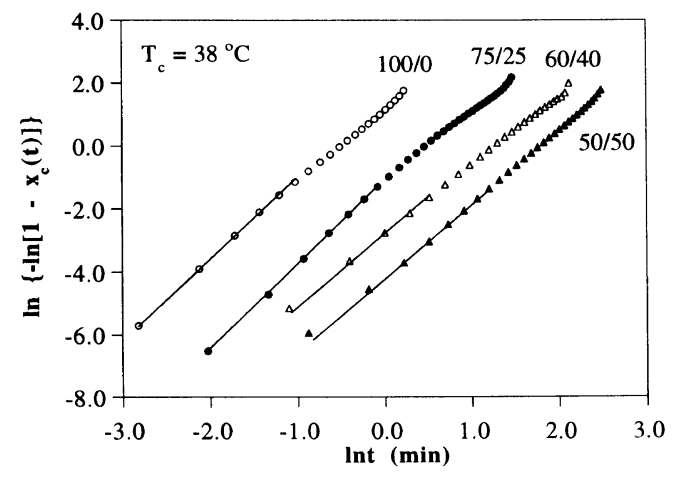

Figure 6. Avrami plots of PCL/PPA blends for crystallization temperature of $38^{\circ} \mathrm{C}$. The corresonding overall blend compositions are shown in the figure.

may thus be ascribed to thinner lamellar thickness formed in PCL-rich phase containing higher PPA content. The presence of PPA imposed some hindrance on the thickening of PCL crystals during crystallization. PCL crystals formed from the matrix containing higher PPA content were consequently thinner and have lower melting points.

Isothermal crystallization kinetics of PCL/PPA blends were also investigated. The crystallization rate was obtained from the Avrami equation:

$$
\ln \left\{-\ln \left[1-x_{\mathrm{c}}(t)\right]\right\}=\ln k_{n}+n \ln t
$$

where $x_{\mathrm{c}}(t)$ is the relative crystallinity, $k_{n}$ is the crystallization rate constant, and $n$ is the Avrami exponent. Relative crystallinity was calculated from the DSC isothermal exotherm as,

$$
x_{\mathrm{c}}(t)=\frac{\int_{0}^{t}(\mathrm{~d} Q / \mathrm{d} t) d t}{\int_{0}^{\infty}(\mathrm{d} Q / \mathrm{d} t) \mathrm{d} t}
$$

where $\mathrm{d} Q / \mathrm{d} t$ is the heat flow of DSC. Figure 6 displays the Avrami plot for various PCL/PPA blends crystallized at $38^{\circ} \mathrm{C}$. Avrami exponent ranges from 2.2 to 2.7 but no systematic variation of $n$ with composition or temperature was found. Therefore, different values of $n$ may arise from experimental error. The crystallization rate constants were determined from the intercepts and were plotted against the overall PCL composition in Figure 7(a) and the PCL composition in PCL-rich phase in

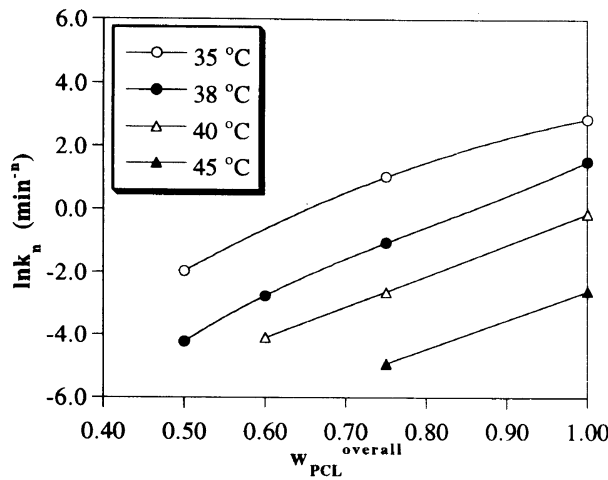

(a)

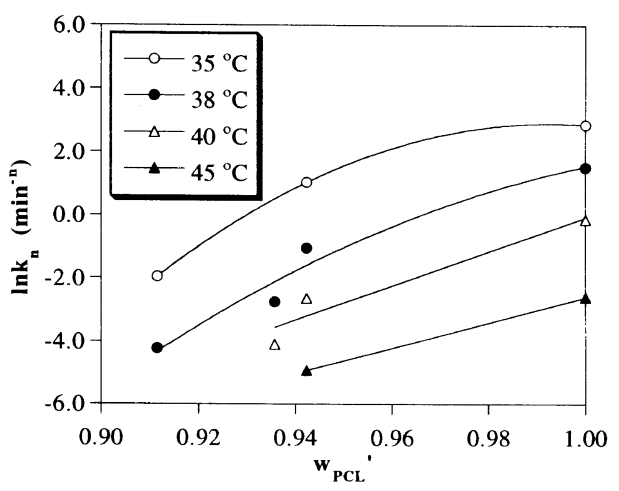

(b)

Figure 7. Variation in crystallization rate constants with (a) overall PCL composition and (b) PCL composition in PCL-rich phase. The corresponding crystallization temperatures are indicated.

Figure 7(b). The crystallization rate increases with overall PCL composition. This is consistent with the suggestion that PCL composition in PCL-rich phase is higher in the blend with more overall PCL content. Of course, in addition to composition, the phase-separated morphology may also affect the crystallization kinetics. The interface between the two phases may provide nucleation sites for crystallization to take place preferentially. Nevertheless, such effect should promote rather than depress the crystallization rate. Since depression in crystallization rate was observed upon blending with PPA, the overall composition effect on the PCL crystallization rate should be governed by the composition of the PCL-rich phase.

The crystallization rate constant in the Avrami equation is a combination of nucleation and crystal growth rates. The growth rate is related to the rate constant by $G \sim k_{n}^{1 / n}$. Using the modification of growth rate by Boon and Azcue for polymer blends by considering an additional entropic contribution due to decreased probability of selecting a crystalline sequence from the miscible melt, the following equation for the rate constants is obtained ${ }^{22}$ :

$$
\begin{aligned}
& \frac{1}{n} \ln k_{n}=\ln k_{p}+\ln \phi_{2}-\frac{U^{*}}{R\left(T_{\mathrm{c}}-T_{\mathrm{o}}\right)} \\
& -\frac{K_{\mathrm{g}}}{T_{\mathrm{c}}\left(T_{\mathrm{mb}}^{0}-T_{\mathrm{c}}\right) f}+\frac{\lambda \sigma T_{\mathrm{mb}}^{0}}{b_{0} \Delta h_{\mathrm{f}}^{0}\left(T_{\mathrm{mb}}^{0}-T_{\mathrm{c}}\right) f} \ln \phi_{2}
\end{aligned}
$$

where $k_{0}$ is a constant, $\phi_{2}$ is the volume fraction of PCL in PCL-rich phase, $U^{*}$ is the activation energy required 


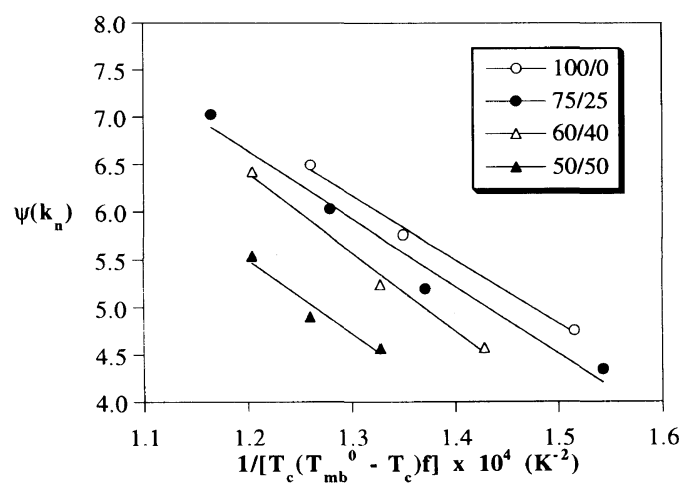

Figure 8. $\psi\left(k_{n}\right)$ vs. $1 /\left[T_{\mathrm{c}}\left(T_{\mathrm{mb}}^{0}-T_{\mathrm{c}}\right) f\right]$ plot of PCL/PPA blends. The overall blend compositions are shown.

Table III. $K_{\mathrm{g}}$ and $\sigma_{\mathrm{e}}$ obtained from Figure 8

\begin{tabular}{ccc}
\hline $\begin{array}{c}\text { Overall PCL/PPA } \\
\text { composition }\end{array}$ & $K_{\mathrm{g}}$ & $\sigma_{\mathrm{c}}$ \\
\hline $100 / 0$ & & 80.8 \\
$75 / 25$ & 67286 & 85.3 \\
$60 / 40$ & 70982 & 100.0 \\
$50 / 50$ & 82914 & 93.6 \\
\hline
\end{tabular}

to transport the segments across the liquid-solid interface, $T_{0}$ is the temperature where such a transport ceases, $T_{\mathrm{mb}}^{0}$ is the equilibrium melting point, $f=2 T_{\mathrm{c}} /$ $\left(T_{\mathrm{mb}}^{0}+T_{\mathrm{c}}\right)$, a factor taking account of the temperature dependence of the enthalpy of melting $\left(\Delta h_{\mathrm{f}}^{0}\right) \lambda$, is a constant, $\lambda=2$ for regimes I and III and $\lambda=1$ for regime II growth, $K_{\mathrm{g}}$ is a secondary nucleation constant given by

$$
K_{\mathrm{g}}=\frac{\alpha b_{0} \sigma \sigma_{\mathrm{e}} T_{\mathrm{m}}^{0}}{k_{\mathrm{B}} \Delta h_{\mathrm{f}}^{0}}
$$

where $\sigma$ and $\sigma_{\mathrm{e}}$ are side and fold surface free energies, respectively; $b_{0}$ is the monomolecular thickness; and $\alpha$ is a constant, $\alpha=4$ for regimes I and III and $\alpha=2$ for regime II growth.

Rearranging eq 14 we otain

$$
\begin{aligned}
\psi\left(k_{n}\right)= & \frac{1}{n} \ln k_{n}-\ln \phi_{2}+\frac{U^{*}}{R\left(T_{\mathrm{c}}-T_{0}\right)} \\
& -\frac{\lambda \sigma T_{\mathrm{mb}}^{0}}{b_{0} \Delta h_{\mathrm{f}}^{0}\left(T_{\mathrm{mb}}^{0}-T_{\mathrm{c}}\right) f} \ln \phi_{2} \\
= & \ln k_{0}-\frac{K_{\mathrm{g}}}{T_{\mathrm{c}}\left(T_{\mathrm{mb}}^{0}-T_{\mathrm{c}}\right) f}
\end{aligned}
$$

Equation 16 prescribes that a plot of $\psi\left(k_{n}\right) v s$. $1 / T_{\mathrm{c}}\left(T_{\mathrm{mb}}^{0}-T_{\mathrm{c}}\right) f$ should yield a straight line with slope given by $K_{\mathrm{g}}$. This analysis was applied to PCL/PPA blends. The universal values of $U^{*}=1500 \mathrm{cal} \mathrm{mol}^{-1}$ and $T_{0}=T_{\mathrm{g}}-30$ were used here for analysis. ${ }^{23} T_{\mathrm{g}}$ of PCL-rich phase was calculated by substituting PCL composition calculated from eq 11 into the Fox equation. Other parameters for analysis include: $\Delta h_{\mathrm{f}}^{0}=1.48 \times 10^{9} \mathrm{erg}$ $\mathrm{cm}^{-3}, b_{0}=4.13 \AA$, and $\sigma=6.1 \mathrm{erg} \mathrm{cm}^{-2}$ as evaluated from Thomas-Staveley equation. ${ }^{23}$ The value of $\lambda$ was taken as unity signifying a regime II crystallization for PCL. ${ }^{23}$
Plots of $\psi\left(k_{n}\right) v s .1 / T_{\mathrm{c}}\left(T_{\mathrm{mb}}^{0}-T_{\mathrm{c}}\right) f$ are shown in Figure 8. Table III lists $K_{\mathrm{g}}$ and $\sigma_{\mathrm{e}}$ obtained for different blend compositions. The fold surface free energy increases slightly with PPA composition. $\sigma_{\mathrm{e}}$ determined by the kinetic analysis is the "kinetic fold surface free energy" related to the difficulty of the chain to perform folding during crystallization. ${ }^{24}$ Increase of $\sigma_{\mathrm{e}}$ with PPA composition suggests that the mixing with PPA imposed greater hindrance on the folding of PCL chains.

\section{CONCLUSIONS}

Blends of PCL with a conjugated polymer, PPA, were studied. PPA and PCL were partially miscible in the melt with the PCL-rich phase containing ca. 91 or $94 \mathrm{wt} \%$ PCL. The melting point decreased upon blending. The isothermal crystallization rate of PCL was depressed by blending due to partial miscibility. The crystal fold surface free energy $\sigma_{\mathrm{e}}$ of $c a .90 \mathrm{erg} \mathrm{cm}^{-2}$ was found from Lauritzen-Hoffman analysis. The fold surface free energy increased slightly with PPA composition, due to hindrance in chain folding by PPA.

\section{REFERENCES}

1. H.-L. Chen, D.-J. Liaw, J.-S. Tsai, J.-S. Shyu, and J.-M. Yang, Polym. J., 28, 976 (1996).

2. A. A. Berlin, L. A. Blumenfeld, M. I. Tscherkachin, A. F. Calmanson, and O. G. Selskaija, Vysokomol. Soedin., 1, 1361 (1959).

3. Y. Okamoto, A. Gordon, F. Movsovicius, H. Hellman, and W. Brenner, Chem. Ind. (London), 2004 (1961).

4. K. Higashiura, K. Tanimoto, N. Hamochi, and M. Oiwa, Kogyo Kagaku Zasshi, 66, 374 (1963).

5. S. Kambara and H. Noguchi, Makromol. Chem., 73, 244 (1964).

6. B. E. Lee and A. M. North, Makromol. Chem., 79, 135 (1964).

7. H. Noguchi and S. Kambara, J. Polym. Sci., B, 1, 533 (1963).

8. R. J. Kern, J. Polym. Sci., A-1, 7, 621 (1969).

9. C. Simionescu and S. Dumitrescu, Makromol. Chem., 135, 47 (1970).

10. I. Negulescu, V. Percec, M. Grigoras, I. Diaconu, M. Leanca, and L. Goras, Vysokomol. Soedin, 16, 790 (1974).

11. P. S. Woon and M. F. Farona, J. Polym. Sci., Polym. Chem. Ed., 12, 1749 (1974)

12. M. Clark and M. F. Farona, Polym. Bull., 7, 445 (1982).

13. T. Masuda, K. Hasegawa, and T. Higashimura, Macromolecules, 7, 728 (1974).

14. K. Hasegawa, T. Masuda, and T. Higashimura, Macromolecules, 8, 255 (1975).

15. T. Masuda, N. Sasaki, and T. Higashimura, Macromolecules, 8 , 717 (1975).

16. T. Masuda, K.-Q. Thieu, N. Sasaki, and T. Higashimura, Macromolecules, 9, 661 (1976).

17. N. Sasaki, T. Masuda, and T. Higashimura, Macromolecules, $\mathbf{9}$, 664 (1976).

18. T. Masuda, Y. Kuwane, K. Yamamoto, and T. Higashimura, Polym. Bull., 2, 823 (1980)

19. T. Masuda, T. Takahashi, K. Yamamoto, and T. Higashimura, J. Polym. Sci., Polym. Chem. Ed., 20, 2603 (1982).

20. D.-J. Liaw, S.-D. Leu, C.-L. Lin, and C.-F. Lin, Polym. J., 24 , 889 (1992).

21. T. G. Fox, Bull. Am. Phys. Soc., 1, 123 (1956)

22. J. Boon and J. M. Azcue, J. Polym. Sci., Part A, 6, 885 (1968).

23. L. Goulet, and R. E. Prud'homme, J. Polym. Sci., Polym. Phys. Ed., 28, 2329 (1990).

24. J. D. Hoffman, G. T. Davis, and J. I. Lauritzen Jr., in "Treatise on Solid State Chemistry," N. B. Hanny, Ed., Plenum Press, New York, N.Y., 1976, Chapter 7. 